Check for updates

Cite this: RSC Adv., 2019, 9, 37518

Received 5th October 2019

Accepted 3rd November 2019

DOI: $10.1039 / c 9 r a 08065 a$

rsc.li/rsc-advances

\section{A high-sensitive room temperature gas sensor based on cobalt phthalocyanines and reduced graphene oxide nanohybrids for the ppb-levels of ammonia detection $\dagger$}

\author{
ZhiJiang Guo, ${ }^{a}$ Bin Wang, (D) *a Xiaolin Wang, ${ }^{b}$ Yong Li, ${ }^{a}$ Shijie Gai, ${ }^{a}$ Yiqun Wu ${ }^{\text {ac }}$ \\ and XiaoLi Cheng ${ }^{a}$
}

Highly sensitive gas sensing materials are of great importance for environmental pollution monitoring. In this study, four nanohybrid materials containing different phenoxyl substituents of cobalt phthalocyanines (tetra- $\beta$-carboxylphenoxylphthalocyanine cobalt (cpoPcCo), tetra- $\beta$-(4-carboxy-3methoxyphenoxy)phthalocyanine cobalt (cmpoPcCo), tetra- $\beta$-phenoxylphthalocyanine cobalt (poPcCo), and tetra- $\beta$-(3-methoxyphenoxy)phthalocyanine cobalt (mpoPcCo)) and reduced graphene oxide $(r \mathrm{GO})$ (RPcCo/rGO) were synthesized via non-covalent interactions as a high performance gas sensing materials for the ppb-level detection of ammonia $\left(\mathrm{NH}_{3}\right)$. Various characterization techniques, including FT-IR, Raman, UV-vis, TGA, XPS and SEM, were used to confirm the structure, element information and morphology of the as-synthesized materials. The obtained materials were used in interdigital electrodes to fabricate the sensing device, and the gas sensing performance was investigated at room temperature. The obtained sensors exhibited excellent sensitivity, selectivity, good reproducibility and perfect response-concentration linearity towards $\mathrm{NH}_{3}$, which are mainly ascribed to the synergetic effects of $\mathrm{RPCCO}$ and $\mathrm{rGO}$ due to the specific surface area structure for $\mathrm{NH}_{3}$ diffusion, the abundant active sites to adsorb $\mathrm{NH}_{3}$, and excellent conductivity for efficient electron transport, particularly the effect of RPcCo. For example, the cpoPcCo/rGO-based sensor showed a higher and faster response for low concentration of $\mathrm{NH}_{3}\left(\sim 2.5\right.$ and $45 \mathrm{~s}$ for $100 \mathrm{ppb}$ of $\left.\mathrm{NH}_{3}\right)$, a ppb level detection and superior stability over 60 days. Besides, the effect of different phenoxyl substituents of cobalt phthalocyanines on the sensing performance and the sensing mechanism for the sensitivity enhancement were discussed and confirmed by the first-principles density functional theory calculations and electrochemical impedance spectroscopy (EIS).

\section{Introduction}

In recent years, various toxic gases (such as $\mathrm{NH}_{3}, \mathrm{CO}_{2}, \mathrm{SO}_{2}$, and $\mathrm{H}_{2} \mathrm{~S}$ ) have been released due to rapid developments and unforeseen accidents in various industries. Hence, a technology to detect gaseous air pollutants and toxic gases has become critical. ${ }^{1}$ Among them, as a highly toxic gas, ammonia $\left(\mathrm{NH}_{3}\right)$ usually comes from the factories, food industries and chemical industries. We know that it is extremely harmful to humans and

${ }^{a}$ Key Laboratory of Functional Inorganic Material Chemistry, Ministry of Education, School of Chemistry and Materials Science, Heilongjiang University, Harbin 150080, P. R. China. E-mail:wangbin@hlju.edu.cn

${ }^{b}$ School of Material and Chemical Engineering, Heilongjiang Institute of Technology, Harbin 150050, P. R. China

'Shanghai Institute of Optics and Fine Mechanics, Chinese Academy of Sciences, Shanghai 201800, P. R. China

$\dagger$ Electronic supplementary information (ESI) available. See DOI: $10.1039 / \mathrm{c} 9 \mathrm{ra} 08065 \mathrm{a}$ the environment. ${ }^{2}$ When the concentration of $\mathrm{NH}_{3}$ exceeds $25 \mathrm{ppm}$, it will cause serious damage to human organs, such as the respiratory tract and eyes and also cause skin irritation., Therefore, it is very important to design and develop ammonia sensing materials with good selectivity, high sensitivity, fast response and recovery performance.

Recently, graphene nanosheets have attracted considerable attention of scientists, particularly graphene oxide (GO) and reduced graphene oxide (rGO). GO and rGO, as twodimensional carbon materials, have a high specific surface area and unique properties, such as high thermal conductivity, good mechanical properties and rich functional groups (such as carboxyl, hydroxyl and epoxy) with excellent chemical stability; hence, they are considered to be promising gas-sensitive materials. ${ }^{5,6}$ In 2004, for the first time, Novoselov et al. found the possibility of inducing charge carriers to graphene by the adsorption of various gases including $\mathrm{H}_{2} \mathrm{O}, \mathrm{NH}_{3}$ and $\mathrm{NO}_{2}{ }^{7}$ Later, in 2007, graphene-based gas sensors were prepared for 
the adsorption of individual gas molecules for detection. ${ }^{8}$ Wehling et al. showed that graphene's peculiar density of states (DOS) was ideal for the "chemical sensor" application and explained the above study. ${ }^{9}$ Leenaerts et al. confirmed that $\mathrm{NO}_{2}$ and $\mathrm{H}_{2} \mathrm{O}$ acted as acceptors, and $\mathrm{CO}$ and $\mathrm{NH}_{3}$ acted as donors by analysing the interactions between different gas molecules and the GO surface using charge transfer analysis. ${ }^{10}$ These experimental and theoretical studies provided a reliable basis for graphene to be considered a good gas-sensitive material. Recently, GO and rGO-based gas sensors have been widely studied as gas sensing materials. Chen et al. showed that GO could detect a series of gas molecules at very low concentrations with detection limits of up to one million molecules. ${ }^{11}$ Li et al. reported that the response of pure rGO was $5.3 \%$ to $50 \mathrm{ppm}$ of $\mathrm{NH}_{3} .{ }^{12}$ Zhou et al. reported that rGO prepared by spray coating showed a response value of less than $5 \%$ for $80 \mathrm{ppm}^{\mathrm{NH}_{3}}$ detection. ${ }^{13}$ In summary, it can be seen that simple graphene has good electrical conductivity and high electromigration. ${ }^{\mathbf{1 4 , 1 5}}$ However, simple GO and rGO show low sensitivity, poor selectivity and slow recovery capability, which does not meet the standard requirements of practical application. ${ }^{\mathbf{1 6}}$

Functional modification is a very reliable way to improve the gas sensing performance of graphite oxide and reduced graphene oxide. For example, doping some heteroatoms in graphene and combining with other gas sensing materials represent a new research method for improving the sensitivity of the hybrid. ${ }^{17}$ An ammonia gas sensor based on polyaniline with N-doped graphene quantum dots was prepared, and showed a response value of 110.92 for $1500 \mathrm{ppm} \mathrm{NH}_{3}{ }^{18}$ Karaduman $e t$ al. reported that the rGO hybrids showed enhanced $\mathrm{NH}_{3}$-sensing properties by the modification of $\mathrm{Ag}$, $\mathrm{Au}$ and $\mathrm{Pt}$ nanoparticles at room temperature. ${ }^{19}$ The $\mathrm{rGO}^{-\mathrm{TiO}_{2}}$ hybridbased sensor has shown excellent sensing properties by improving the morphology of rGO sheets due to the introduction of synthetic $\mathrm{TiO}_{2} \cdot{ }^{20}$ However, it is still a big challenge for GO and rGO hybrids to obtain excellent sensitivity, selectivity, and good stability.

It is well-known that metal phthalocyanines (MPcs) are also good gas sensitive materials due to their unique conjugated $18 \pi$-electron structure. ${ }^{21}$ There are 16 hydrogen atoms around the phthalocyanine ring, which can be substituted by other groups, such as alkoxy group, carboxyl group, amino group and sulfonic acid group ${ }^{22-24}$ which provides an opportunity to improve and optimize the $\mathrm{NH}_{3}$-sensing performance of rGO by using substituted MPcs as dopants. In addition, there is a hole in the ring that can accommodate a variety of different metal elements to form different kinds of metal phthalocyanines. This structure is conducive to further optimize the $\mathrm{NH}_{3}$-sensing performance due to highly active central metal atoms for selective $\mathrm{NH}_{3}$ adsorption. More importantly, the unique planar $18 \pi$-electron-conjugated structure of MPcs allows them to be closely coupled with the rGO by $\pi-\pi$ interactions. ${ }^{25}$ On the one hand, the MPcs provide more gas sensing active sites for selective $\mathrm{NH}_{3}$ adsorption. On the other hand, rGO provides fast and efficient charge transfer. ${ }^{26,27}$ Apparently, the $\mathrm{NH}_{3}$-sensing performance of $\mathrm{MPc} / \mathrm{rGO}$ hybrids will be greatly improved. ${ }^{28}$
However, MPcs and rGO hybrids have rarely been reported as gas sensing materials.

Based on the above consideration, herein, four nanohybrid materials containing different phenoxyl substituents of cobalt phthalocyanines (tetra- $\beta$-carboxylphenoxylphthalocyanine cobalt (cpoPcCo), tetra- $\beta$-(4-carboxy-3-methoxyphenoxy) phthalocyanine cobalt (cmpoPcCo), tetra- $\beta$-phenoxylphthalocyanine cobalt (poPcCo), and tetra- $\beta$-(3-methoxyphenoxy) phthalocyanine cobalt (mpoPcCo)) coupled with reduced graphene oxide (RPcCo/rGO) were successfully prepared (see Scheme 1). The effect of the substituents of cobalt phthalocyanines on gas sensing performance was investigated. The presence of different substituents on the phthalocyanine greatly affects the gas sensitivity of the RPcCo/rGO hybrids. When the electron-withdrawing carboxyl group is present on the phenoxyl substituents of the phthalocyanine ring, it increases the hole sites of the phthalocyanine and provides a lot of active sites for $\mathrm{NH}_{3}$ adsorption. Therefore, the cpoPcCo/rGO hybrid exhibited a high gas response of $42.4 \%$ for 100 ppm of $\mathrm{NH}_{3}$ with a reversible recovery time of $120 \mathrm{~s}$ and a very low detection limit of $3.7 \mathrm{ppb}$. Furthermore, the selectivity, stability and the $\mathrm{NH}_{3}$ sensing mechanism have also been studied in detail.

\section{Experimental and calculation details}

\subsection{Materials and reagents}

Flake graphite was purchased from Shenzhen Nanotech Port Co. Ltd. 4-Nitrophthalonitrile (99\% purity), phenol (98\% purity), methoxyphenol (98\% purity), p-hydroxybenzoic acid (99.5\% purity), sodium 4-hydroxy-3-methoxybenzoate (>98\% purity) and DBU (98\% purity) were purchased from SigmaAldrich Co. LLC. Ultrapure water (resistivity $18.2 \mathrm{M} \Omega \mathrm{cm}$ ) was obtained from a Milli-Q Water System (Millipore Corp., Bedford, MA, USA) and was used throughout the experiments. Graphene oxide (GO) was fabricated using the modified Hummers' method from graphite powder, which was described in our former reports. ${ }^{29}$ Tetra- $\beta$-carboxyphenoxyphthalocyanine cobalt(II) (cpoPcCo) was synthesized by the universal template

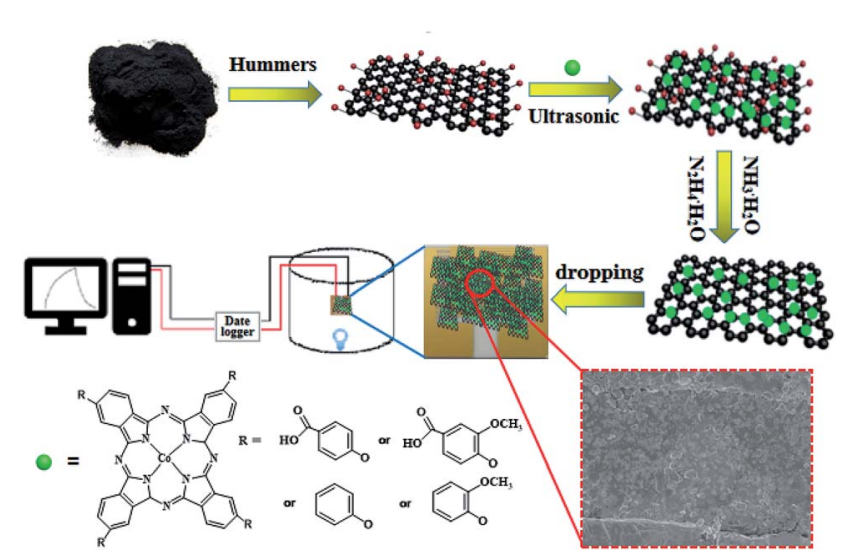

Scheme 1 Synthesis and processing schematic of RPcCo/rGO hybrids and gas sensor device. 
reaction of 4-(4-carboxyphenoxy) phthalonitrile with anhydrous cobalt(II) chloride in the presence of 1,8-diazabicyclo[5,4,0] undec-7-ene (DBU). The other cobalt phthalocyanine(II) is compounded similarly and only 4-(4-carboxyphenoxy)phthalonitrile is replaced (Scheme S1, see Experiment details in ESI†). All other reagents were of analytical grade and utilized without further purification.

\subsection{Preparation of PcCo/rGO hybrids}

Four RPcCo/rGO hybrids, poPcCo/rGO, mpoPcCo/rGO, cpoPcCo/rGO, cmpoPcCo/rGO, were prepared by the uniform method: GO $(0.100 \mathrm{~g})$ was sonicated in $N, N$-dimethylformamide (DMF) solution $(30 \mathrm{~mL})$ at room temperature for $4 \mathrm{~h}$. The corresponding PcCo $(0.200 \mathrm{~g})$ was dissolved in DMF solution (10 $\mathrm{mL}$ ), and then the PcCo solution was added to GO solution dropwise. The ensuing mixture was sonicated for further $48 \mathrm{~h}$ at room temperature. Then, hydrazine hydrate $(0.6 \mathrm{~mL})$ and ammonia $(4 \mathrm{~mL})$ were added and stirred at $100{ }^{\circ} \mathrm{C}$ for $24 \mathrm{~h}$ under a nitrogen atmosphere. After cooling, the resultant solution was filtered through a $0.20 \mu \mathrm{m}$ Millipore, and washed successively by DMF, ethanol and acetone until the filtrate was colourless. Subsequently, the resultant product was dried in a vacuum at $60{ }^{\circ} \mathrm{C}$ for $5 \mathrm{~h}$. In addition, rGO was prepared using the same procedure as used for $\mathrm{RPcCo} / \mathrm{rGO}$ hybrids but without the addition of RPcCo, and RPcCo covalently linked rGO (RPcCorGO) was also prepared using a method reported in literature. ${ }^{30}$

\subsection{Sensor assembling and sensing measurements}

An entire description of the gold electrodes and the gas sensor testing device was illustrated in our previous research, (the detailed structure parameters for gas sensors see Experiment details in ESI $\dagger)^{29,31}$

To prepare gas sensors composed of $\mathrm{RPcCo} / \mathrm{rGO}$ hybrids, the as-prepared $\mathrm{RPcCo} / \mathrm{rGO}$ hybrids were dispersed in ethanol to attain a uniform suspension of $1.0 \mathrm{mg} \mathrm{mL}^{-1}$ ultrasonication for $2 \mathrm{~h}$, and then about $50 \mu \mathrm{L}$ of dispersion was dropped on the interdigitated electrodes using a microsyringe. After the evaporation of the solvent, any residue of the solvent left was thoroughly removed by drying the sensor devices in a vacuum oven for $2 \mathrm{~h}$ at $80^{\circ} \mathrm{C}$. By contrast, the gas sensors of $\mathrm{rGO}$ and RPcCorGO were also fabricated by the similar procedures.

A classic sensing test cycle composed of three sequential steps to speed up the recovery of the sensor, a mini ultraviolet (UV) lamp (10 V/3 W, $254 \mathrm{~nm})$ was used to removal of the gas. First, airflow was introduced into the sensing test chamber to obtain a baseline, and then the chamber was vacuumed by the pump. Later, a standard gas was injected to the chamber by a syringe, and then $\mathrm{RPcCo} / \mathrm{rGO}$ hybrid sensor was placed in testing gas to register sensor signals. Finally, the sensor was recovered in the air under UV light. All measurements were performed at $29{ }^{\circ} \mathrm{C} \pm 0.5{ }^{\circ} \mathrm{C}$ with a relative humidity of $55 \% \pm 5 \%$. In this study, sensitivity $(S)$ is defined by the relative resistance change, as follows:

$$
\text { Response }(\%)=\frac{\Delta R}{R_{\mathrm{a}}} \times 100 \%=\frac{R_{\mathrm{g}}-R_{\mathrm{a}}}{R_{\mathrm{a}}} \times 100 \%
$$

where $R_{\mathrm{a}}$ is the sensor resistance in the initial airflow, which was used as the background and $R_{g}$ is the sensor resistance after being exposed to a certain concentration of target gas. Response and recovery times are defined as the time needed for $90 \%$ of total resistance change on exposure to the target gas and air, respectively. The sensor responses to different $\mathrm{RH}$ were measured at $29^{\circ} \mathrm{C} \pm 0.5^{\circ} \mathrm{C}$, and a certain $\mathrm{RH}$ level was achieved by mixing dry air and water vapour. ${ }^{23}$

\subsection{Characterization}

UV/vis absorption spectra were recorded using a Lambda $35 \mathrm{UV} /$ vis spectrometer (PerkinElmer, USA). FT-IR spectra were recorded on a Nicolet FT-IR NEXUS spectrometer (Thermo Scientific). The Raman spectra were obtained using a Raman spectrophotometer (HR800, HORIBA JobinYvon Company) exploited by a laser with a $457.9 \mathrm{~nm}$ wavelength. Scanning electron microscopy (SEM) images were recorded using a Hitachi S-4800 field emission scanning electron microscope, operating at $15 \mathrm{kV}$. Transmission electron microscope (TEM) images were recorded on a JEM-3010 electron microscope (JEOL, Japan) at an acceleration voltage of $300 \mathrm{kV}$. X-ray photoelectron spectroscopy (XPS) measurements were performed using AXIS UL TRA DLD. Thermogravimetric (TG) analysis was performed on TA Q600 under a stream of nitrogen at a heating rate of $10{ }^{\circ} \mathrm{C} \mathrm{min}^{-1}$.

\subsection{Calculation details}

DFT calculations were performed for the adsorption of $\mathrm{NH}_{3}$ on RPcCos, using long-range corrected functional of CAM-B3LYP with a set of hybrid basis sets (LanL2DZ for metals and 6$31 \mathrm{G}(\mathrm{d})$ for $\mathrm{H}, \mathrm{C}$, and $\mathrm{N}){ }^{32}$ Charge analyses were performed with the NBO (natural bond orbital) method. ${ }^{33}$ The Gaussian 09 quantum chemical package was employed for all calculations in the present study. ${ }^{34}$

\section{Results and discussion}

\subsection{Characterization of RPcCo/rGO hybrids}

The absorbance spectrum represents an important part of the UV-vis spectrum of phthalocyanine complexes. Fig. $1 \mathrm{~A}$ and $\mathrm{S} 2 \dagger$ show the UV-vis spectra of rGO, RPcCos and RPcCo/rGO in DMF. cpoPcCo, cmpoPcCo, poPcCo and mpoPcCo display maximum peaks at 662, 665, 664 and $668 \mathrm{~nm}$, respectively, which are the characteristic absorptions of phthalocyanine in the Q-band region at around $650-700 \mathrm{~nm}$, attributed to the $\pi-$ $\pi^{*}$ transitions from the HOMO (highest occupied molecular orbital) to the LUMO (lowest unoccupied molecular orbital) of the $\mathrm{Pc}^{2-}$ ring. ${ }^{35}$ Because of the $\pi-\pi$ interactions, RPcCo could spontaneously assemble with $\mathrm{rGO}$, generating $\mathrm{RPcCo} / \mathrm{rGO}$ hybrids. As shown in Fig. $1 \mathrm{~A}$ and $\mathrm{S} 2, \uparrow$ the characteristic absorption in the Q-band of RPcCo is observed in the spectra of $\mathrm{RPcCo} / \mathrm{rGO}$ hybrid. Moreover, the RPcCo/rGO hybrids were further confirmed by the disappearance of the characteristic absorption of RPcCo in the absorbance spectrum of rGO. In addition, compared with the Q-band absorption of RPcCo, the Q-band absorption of the $\mathrm{RPcCo} / \mathrm{rGO}$ hybrids red-shifted and broadened due to the reduction in the HOMO-LUMO energy 

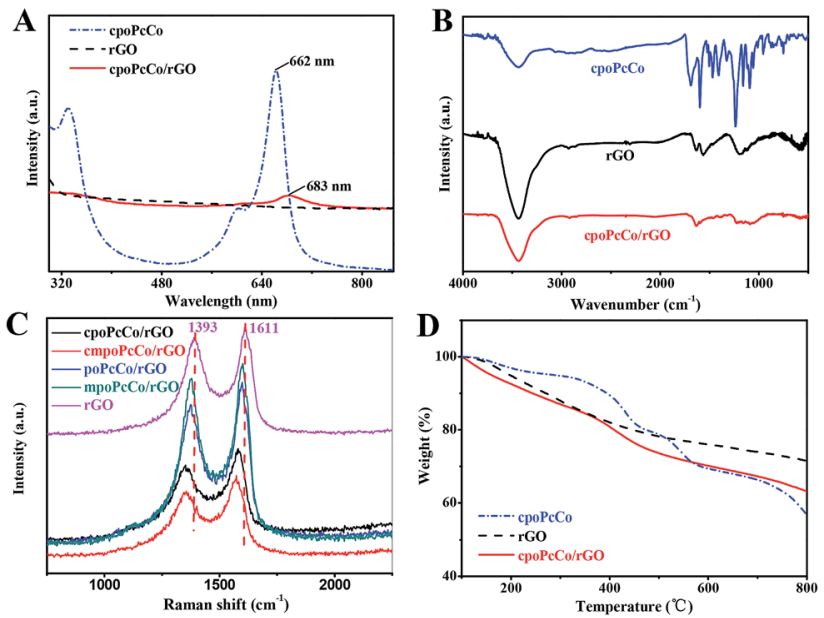

Fig. 1 (A) UV-vis spectra of $\mathrm{rGO}, \mathrm{cpoPcCo}$ and $\mathrm{cpoPcCo} / \mathrm{rGO}$ hybrids in DMF; (B) FT-IR spectra of rGO, cpoPcCo and cpoPcCo/rGO hybrids; (C) Raman spectra of $\mathrm{rGO}, \mathrm{RPcCo} / \mathrm{rGO}$ hybrids obtained at $\lambda_{\mathrm{exc}}=$ $457.9 \mathrm{~nm}$; (D) TG profiles of rGO, cpoPcCo and cpoPcCo/rGO hybrid.

gap of the phthalocyanine ring by $\pi-\pi$ interactions between the Pc and rGO. ${ }^{36,37}$ Moreover, the $\mathrm{Q}$ band absorption of the substituted MPcs red-shifted in comparison with that of unsubstituted MPcs, because of the reduction in the HOMOLUMO energy gap of the Pc ring by the introduction of the substituents. ${ }^{35}$ In the case of the presence of different substituents on the Pc ring, these band absorption peaks are different due to the electron-withdrawing and donating properties of the substituents. The Q-band of cmpoPcCo red-shifted (3 $\mathrm{nm})$ in comparison with that of cpoPcCo due to the electron-donating properties of the methoxy substituent and was blue-shifted (3 $\mathrm{nm}$ ) in comparison with that of mpoPcCo due to the electronwithdrawing properties of the carboxyl substituent. Because of the existence of lone pair electrons in $\mathrm{NH}_{3}$, the response interactions between $\mathrm{NH}_{3}$ and carboxyl-substituted PcCo could be increased due to the electron-withdrawing properties of the carboxyl substituent on the Pc ring, thus helping to increase its overall ammonia sensitivity.

The successful combination of RPcCos to rGO to give RPcCo/ rGO hybrids was verified by FTIR. Fig. $1 \mathrm{~B}$ and S3† show the FTIR spectra of RPcCo, rGO and RPcCo/rGO hybrids. The spectrum of the cpoPcCo/rGO hybrid (Fig. 1B) shows the characteristic vibrations at $1636 \mathrm{~cm}^{-1}$ and $3440 \mathrm{~cm}^{-1}$ for $\nu \mathrm{C}=\mathrm{C}$ and $\nu \mathrm{O}-\mathrm{H}$ of $\mathrm{rGO}$, respectively, while the characteristic fingerprints of cpoPcCo bands were also present at $1000-1600 \mathrm{~cm}^{-1}$, thus providing a significant support for the formation of cpoPcCo/ rGO hybrid..$^{30,38,39}$ The cmpoPcCo/rGO, poPcCo/rGO, and mpoPcCo/rGO hybrids (Fig. S3†) show very similar FT-IR spectra with the cpoPcCo/rGO hybrid. A significant $\pi$-electron interaction between the donor PcCo and the acceptor rGO basal plane was further confirmed by Raman spectroscopy. As shown in Fig. 1C, there are two characteristic bands at about $1393 \mathrm{~cm}^{-1}$ (D-band) and $1610 \mathrm{~cm}^{-1}$ (G-band) in the Raman spectrum of rGO. Compared with those of rGO, both the D- and G-bands of the RPcCo/rGO hybrids were shifted to lower wavenumbers due to the $\pi-\pi$ conjugated interactions between RPcCo and rGO, the increase in charge carrier abundance provided by the PcCo molecules on the graphene-based surface, which ultimately increases the Fermi level, ${ }^{40}$ well-consistent with the ZnPc-GO hybrid. ${ }^{41}$ In addition, the order of the blueshift of the $\mathrm{PcCo} /$ rGO complex from small to large is cpoPcCo/rGO, cmpoPcCo/ rGO, poPcCo/rGO and mpoPcCo/rGO. The blueshift increased with the increase in the electron-withdrawing properties of the substituent on the Pc ring. This has a great relationship with the charge transfer rate between PcCo and rGO and the effect of different substituents on the metal phthalocyanine on the complex.

The amount of cpoPcCo assembled on rGO was evaluated by TG analysis under $\mathrm{N}(\mathrm{g})$ atmosphere, as shown in Fig. 1D. The weight loss in rGO and cpoPcCo was about $28.48 \%$ and $43.20 \%$ in the range of $100-800{ }^{\circ} \mathrm{C}$, respectively, due to the decarboxylation of the rGO surface oxide species, destruction of residual amorphous carbon and decomposition of cpoPcCo. In the same temperature range, the cpoPcCo/rGO hybrid revealed a $36.85 \%$ weight loss. From the weight loss of rGO, the corrected weight loss caused by cpoPcCo was estimated to be $8.37 \%$. In fact, considering the weight loss of cpoPcCo and rGO, the amount of cpoPcCo adsorbed on the surface of rGO can be calculated as an actual ratio of 19.37 (8.37\%/43.20\%). Simultaneously, TG analysis images of cpoPcCo/rGO, poPcCo/rGO and mpoPcCo/rGO complexes were obtained, as shown in Fig. S4. $\dagger$ The amount of cmpoPcCo, poPcCo and mpoPcCo assembled on rGO were $16.02 \%, 21.04 \%$ and $18.61 \%$, respectively. These results also demonstrate the successful preparation of the $\mathrm{RPcCo} / \mathrm{rGO}$ hybrids. It can also be seen that the amount of RPcCo complex adsorbed on rGO was very similar.

XPS was also conducted to investigate the as-prepared surface chemical composition of RPcCo/rGO hybrids. As can be seen in Fig. 2, the distinct $\mathrm{N}$ 1s and Co 2p peaks emerge in the survey spectra of cpoPcCo/rGO hybrids compared to that of

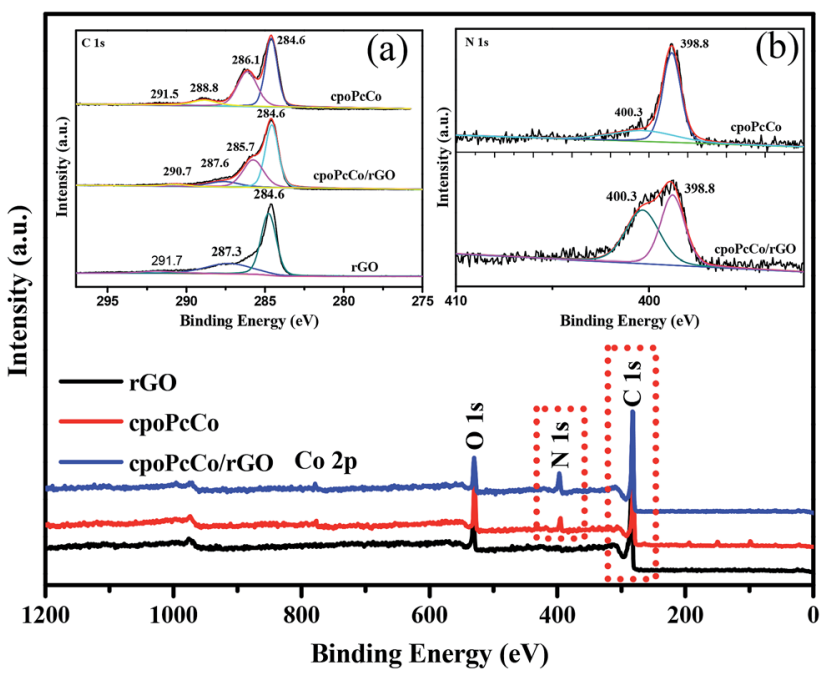

Fig. 2 XPS full survey spectra of $r G O, c p o P c C o$ and cpoPcCo/rGO hybrid; the inset images of (a) and (b) are the high-resolution XPS spectrum of $\mathrm{C} 1 \mathrm{~s}$ and $\mathrm{N}$ 1s of the $\mathrm{rGO}, \mathrm{cpoPcCo}$ and cpoPcCo/rGO hybrids. 
rGO. In the high-resolution C 1s XPS spectrum of cpoPcCo/rGO hybrid (the inset image of (a) in Fig. 2), the peak for cpoPcCo, rGO situated $291.5 \mathrm{eV}, 291.7(\mathrm{C}=\mathrm{O})$ shifts to $290.7 \mathrm{eV}$, the peak for cpoPcCo, rGO situated $288.8 \mathrm{eV}, 287.3(\mathrm{C}-\mathrm{OH})$ shifts to $287.6 \mathrm{eV}$. Compared with the C 1s XPS spectrum of rGO, the new peak situated $286.1 \mathrm{eV}(\mathrm{C}-\mathrm{N})$ was derived form cpoPcCo, which appears and shifts to $285.7 \mathrm{eV}^{30,39}$ In addition, the peaks at $398.7 \mathrm{eV}$ (aza bridge nitrogen) and $400.1 \mathrm{eV}$ (pyrrole nitrogen) can be observed in the high-resolution N 1s XPS spectrum of the cpoPcCo/rGO hybrid (the inset image of (b) in Fig. 2), which was also derived form cpoPcCo. The XPS spectrum of the cpoPcCo/ rGO hybrid contains all the peaks, corresponding to the cpoPcCo and rGO. Similar results can be observed in XPS spectra of others the RPcCo/rGO hybrids (Fig. S5-S7†). All of this evidence further suggests that RPcCo is successfully combined with the rGO.

\subsection{Gas sensing properties}

The surface morphology of the RPcCo/rGO hybrid prepared on the interdigitated electrode was investigated by SEM, as shown in Fig. 3 and S8. $\dagger$ It can be clearly seen that RPcCo/rGO hybrids display a few-layered flake like shape with a rounded morphology. Fig. 3 shows that cpoPcCo/rGO sheets are evenly, loosely distributed between the two-finger of the interdigitated electrode, which provides continuous conducting pathways for the transportation of electrons, the permeable channels for the diffusion of gas molecules, and more advantages on the active sites, giving rise to the high sensing performance. ${ }^{42,43}$

The gas sensing properties of RPcCos-based, rGO-based and $\mathrm{RPcCo} / \mathrm{rGO}$ hybrid-based sensors deposited onto the interdigitated electrode were investigated by using ammonia as the target gas. The selectivity is an important characteristic for the application of sensors at room temperature. The response of nine sensors to $\mathrm{NH}_{3}$ gas with a concentration of $100 \mathrm{ppm}$ and 17 different gases with a concentration of $5000 \mathrm{ppm}$ was investigated, and the results are shown in Fig. 4A. The RPcCo/rGO hybrid-based sensors exhibited the highest response to $\mathrm{NH}_{3}$, particularly the cpoPcCo/rGO hybrid to $100 \mathrm{ppm}$ of $\mathrm{NH}_{3}$ (42.4). The RPcCo/rGO hybrids showed the excellent selectivity toward $\mathrm{NH}_{3}$ as opposed to other gases. As for other sensors, the PcCos was not high enough, but showed a relatively good selectivity to $\mathrm{NH}_{3}$. On the contrary, the rGO sensor showed a poor response and selectivity to $\mathrm{NH}_{3}$, which was consistent with the reported literature (Fig. 4A and $\mathrm{S} 9 \dagger$ ). ${ }^{44}$ The results indicated that the introduction of RPcCo could significantly enhance the response
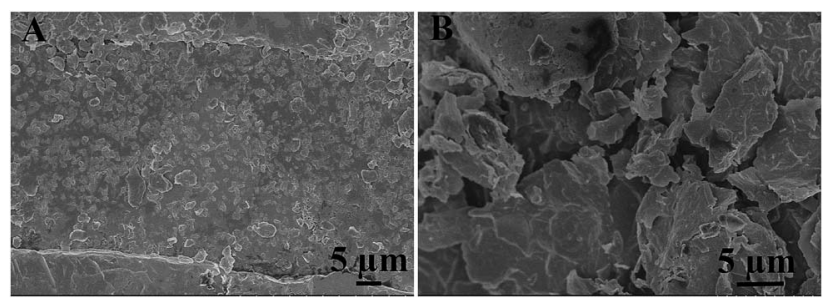

Fig. 3 SEM images of cpoPcCo/rGO hybrids on the interdigital electrode, respectively.

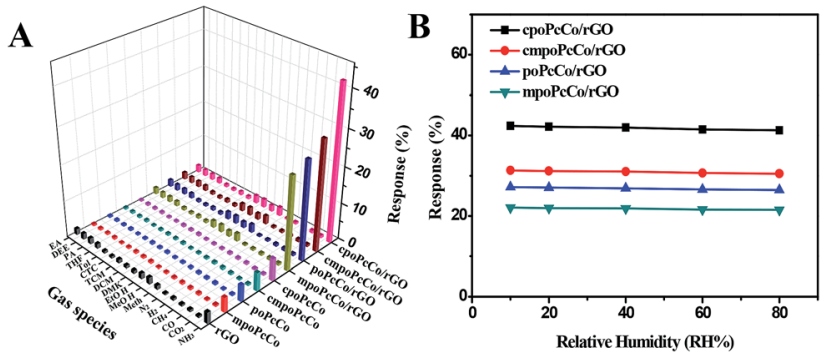

Fig. 4 (A) Response of RPcCos, $\mathrm{rGO}$ and RPcCo/rGO hybrid-based sensors to $100 \mathrm{ppm}$ of $\mathrm{NH}_{3}$ and $5000 \mathrm{ppm}$ of others various gas, respectively. (B) Response of RPcCo/rGO hybrid-based sensors to $100 \mathrm{ppm} \mathrm{NH}$ in different relative humidity at $29^{\circ} \mathrm{C}$.

and selectivity to $\mathrm{NH}_{3}$ due to strong interactions between the central metal of RPcCo and $\mathrm{NH}_{3} \cdot{ }^{45}$ The effect of humidity on sensors is also an important factor for the application of sensors at room temperature. So, the response of the $\mathrm{RPcCo} / \mathrm{rGO}$ hybrid sensors to $100 \mathrm{ppm}$ of $\mathrm{NH}_{3}$ was tested under different relative humidities at $29^{\circ} \mathrm{C}$. As shown in Fig. 4B, the response of RPcCo/ rGO hybrid sensors to $100 \mathrm{ppm} \mathrm{NH}_{3}$ was almost unchanged in different $\mathrm{RH}$, which indicated that the as-prepared material possessed strong stability in a practical environment. Based on the results discussed above, the RPcCo/rGO hybrid sensors display superior selectivity and low effect of RH in its sensing response. Therefore, the $\mathrm{RPcCo} / \mathrm{rGO}$ hybrids are promising candidates for use as a $\mathrm{NH}_{3}$ sensing material.

To further investigate the $\mathrm{NH}_{3}$ sensing properties of $\mathrm{RPcCo} /$ rGO hybrid sensors, the signal resistor of the four RPcCo/rGO hybrid-based sensors were exposed to different concentrations of $\mathrm{NH}_{3}$, the signal resistor was continuously recorded, and then
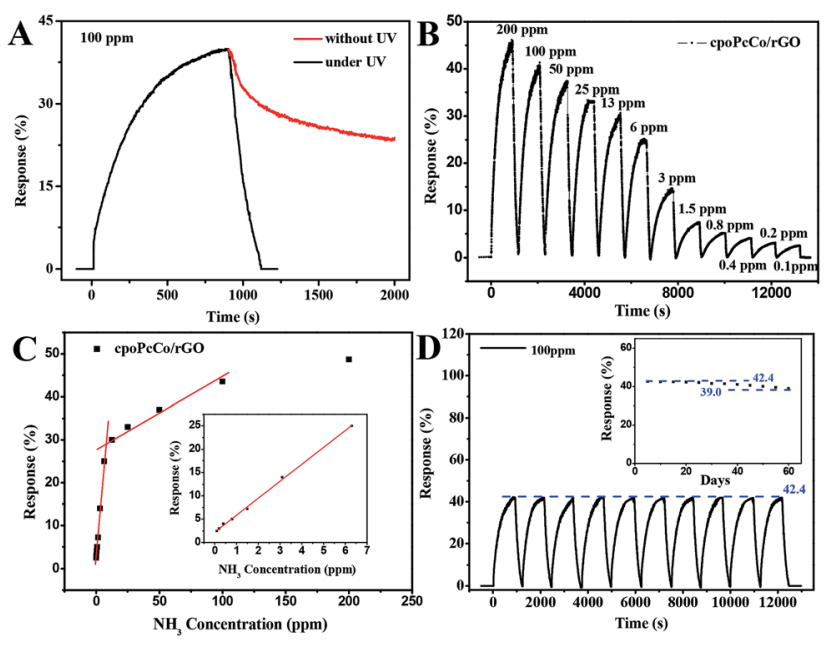

Fig. 5 (A) Response of the cpoPcCo/rGO hybrid sensor exposure to $100 \mathrm{ppm} \mathrm{NH}$ and recovery under UV light or without UV light; (B) resistance of the cpoPcCo/rGO hybrid sensor upon exposure to varying concentrations of $\mathrm{NH}_{3}$; (C) relationship of the response of the cpoPcCo/rGO hybrid sensor to the concentration $\mathrm{NH}_{3}$; (D) ten sensing cycles of the cpoPcCo/rGO hybrid sensor to $100 \mathrm{ppm} \mathrm{NH}_{3}$ (inset: the reproducibility characteristics of the $\mathrm{cpoPcCo} / \mathrm{rGO}$ hybrid sensor to $100 \mathrm{ppm} \mathrm{NH}$ within 60 days) at $29^{\circ} \mathrm{C}$. 
recovered under UV light at room temperature. As shown in Fig. 5A, the recovery time of cpoPcCo/rGO was about $180 \mathrm{~s}$ with a concentration of $100 \mathrm{ppm} \mathrm{NH}_{3}$ under UV light, but the recovery property was very poor without UV light. It was found that the recovery of the cpoPcCo/rGO hybrid strongly depends on UV assistance, which was consistent with other RPcCo/rGO hybrids in Fig. S10-S12. $\dagger$ Under UV irradiation, lightgenerating electrons can react with $\mathrm{O}_{2}$ in the air to produce adsorbed oxygen, and then the remaining holes are formed, so the resistance of the $\mathrm{PcCo} / \mathrm{rGO}$ complex is lowered and the sensor is recovered..$^{30,46}$ Further, twelve dynamic response and recovery cycles were tested exposing to the $\mathrm{NH}_{3}$ concentration from $100 \mathrm{ppb}$ to $200 \mathrm{ppm}$ (Fig. 5B). Clearly, the cpoPcCo/rGO sensor exhibited a stable and reversible response signal towards $\mathrm{NH}_{3}$, even at a low concentration of $\mathrm{NH}_{3}(100 \mathrm{ppb})$. Moreover, it can be observed that the response gradually increased as the target gas concentration increased from $100 \mathrm{ppb}$ to $200 \mathrm{ppm}$. The response of the cpoPcCo/rGO sensor with the concentration of $\mathrm{NH}_{3}$ exhibits two good linear responses, as shown in Fig. 5C, 3.63\% per ppm $\mathrm{NH}_{3}$ for concentration ranging from $0.1-6 \mathrm{ppm}$, and $0.14 \%$ per $\mathrm{ppm}$ $\mathrm{NH}_{3}$ for concentration ranging from 12-100 ppm. A linear regression equation of $S=3.63 C(\mathrm{ppm})+2.23\left(R^{2}=0.998\right)$ was derived from the calibration curve. Reproducibility and stability are very important factors in evaluating gas sensors. The cycling stability and long-term stability towards $100 \mathrm{ppm} \mathrm{NH}_{3}$ were measured at the room temperature. After 10 cycles, the cpoPcCo/rGO sensor still maintained a fast response and recovery capability, and the response showed no difference, as shown in Fig. 5D. In addition, the response changes only 8.0\% during sixty days, which also shows that the cpoPcCo/rGO sensor has excellent long-term stability towards $\mathrm{NH}_{3}$. The $\mathrm{NH}_{3}$ sensing performance of other three $\mathrm{RPcCo} / \mathrm{rGO}$ sensors were conducted and are shown in Fig. S10-S12, $\dagger$ which also show high sensitivity, good linear response, long-term stability and reproducibility, which are also significantly superior to the previously reported sensors (as shown in Table S1 ). Moreover, in order to investigate the effect of cobalt phthalocyanine content on the sensing performances based on the rGO-based hybrids, the cpoPcCo/rGO2 and cpoPcCo/rGO3 were prepared by sonicating GO $(0.100 \mathrm{~g})$ DMF solution with cpoPcCo $(0.100 \mathrm{~g})$ and cpoPcCo $(0.300 \mathrm{~g})$ DMF solution. The response of cpoPcCo/ rGO2 and cpoPcCo/rGO3 sensors upon varying the concentration of $\mathrm{NH}_{3}$ was measured. Fig. $\mathrm{S} 13 \uparrow$ shows that the response of the cpoPcCo/rGO2 and cpoPcCo/rGO3 sensors increase as the concentration of $\mathrm{NH}_{3}$ increases, which is consistent with the characteristics of the cpoPcCo/rGO sensor. Moreover, it is clear that the cpoPcCo/rGO sensor exhibits the highest response (42.5\% to $\left.100 \mathrm{ppm} \mathrm{NH}_{3}\right)$.

\subsection{Gas sensitivity mechanism}

The reasons for the excellent gas sensing performance of $\mathrm{RPcCo} / \mathrm{rGO}$ sensors can be ascribed to the synergistic effects of RPcCo and rGO. The schematic of the RPcCo/rGO hybrid gassensing mechanism for $\mathrm{NH}_{3}$ is exhibited in Fig. S14a. $\dagger$ First, when RPcCo/rGO sensors are exposed to ambient air, the oxygen molecules can rapidly and spontaneously adsorb on the center metal atoms of RPcCo, and form adsorbed oxygen species $\left(\mathrm{O}_{2}{ }^{-}\right)$by further capturing electrons from the conduction band of RPcCo. ${ }^{39,47,48}$ When the RPcCo/rGO sensors were exposed to reducing gases (for instance, $\mathrm{NH}_{3}$ ), these gas molecules reacted with the adsorbed oxygen species, as indicated in the following equation: $4 \mathrm{NH}_{3}(\mathrm{~g})+3 \mathrm{O}_{2}{ }^{-}$(ads) $\rightarrow 2 \mathrm{~N}_{2}(\mathrm{~g})+6 \mathrm{H}_{2} \mathrm{O}$ (g) $+6 \mathrm{e}^{-} .{ }^{39}$ This process releases the electrons, and then rapidly transfer to the rGOs as the electron transfer energy barrier between RPcCo and rGO nanomaterial is low. Fig. S14b-e† show the optical absorption spectra of cpoPcCo and rGO, from which the band gaps of cpoPcCo and rGO can be estimated from the plot of $(A h \nu)^{2}$ (for direct band gap) versus photon energy $(h \nu)$. The intercepts of the tangent to the $x$-axis give a good approximation of the band gap of cpoPcCo and rGO to be 1.83 and $1.13 \mathrm{eV}$, respectively. It indicates that cpoPcCo has lower conductivity compared to highly conductive rGO. Moreover, cpoPcCo strongly interacts with rGO via $\pi-\pi$ interactions. Thus, more electrons transfer from cpoPcCo to rGO due to the higher surface area of the cpoPcCo/rGO hybrids. Finally, the holes in the P-type rGOs recombined with electrons, which lead to an increase in resistance. Contrary to the response process, the $\mathrm{RPcCo} / \mathrm{rGO}$ hybrids can lose electrons quickly by reacting with the reappearing oxygen molecules in the desorption process of ammonia gas, which promotes the sensor resistance to rapidly return to the baseline position. The response of four RPcCo/rGO sensors increases as the concentration of $\mathrm{NH}_{3}$ increases, which is the characteristic of P-type semiconductors. To verify the mechanism, the signal resistors of the RPcCo/rGO sensors were investigated when it is exposed to strong oxidants, e.g., $\mathrm{NO}_{2}$ (Fig. S15†). Contrary to $\mathrm{NH}_{3}$, the resistance reduces sharply when $\mathrm{RPcCo} / \mathrm{rGO}$ sensors are exposed to $\mathrm{NO}_{2} \cdot \mathrm{NO}_{2}$ can trap the electrons from RPcCo/rGO hybrids and obtain more holes in hybrids, leading to the reduced resistance (see Fig. S15†), which is consistent with the charge-transfer mechanism. Moreover, the response of the cpoPcCo/rGO sensor to $10 \mathrm{ppm} \mathrm{NO}_{2}$ is about $1.5 \%$ which is far less than the response of the cpoPcCo/rGO sensor to $\mathrm{NH}_{3}$, and the recovery characteristic is also poor. The result indicates that the cpoPcCo/rGO hybrid has an excellent selectivity toward $\mathrm{NH}_{3}$.

To verify the above-mentioned mechanism, the cpoPcCorGO and cmpoPcCo-rGO hybrids were successfully synthesized based on covalent bonding, as characterized by its UV-vis, FTIR spectra and TG analysis (Fig. S16 and S17 $\dagger$ ). However, the $\mathrm{NH}_{3}$ sensing response of the cpoPcCo-rGO and cmpoPcCo-rGO hybrids was less than that of the cpoPcCo/rGO and cmpoPcCo/rGO hybrids (Fig. S18 $\dagger$ ). These results indicate that the non-covalent strategy is more advantageous, which is mainly ascribed to the synergetic effect of RPcCo and rGO due to the large-surface-area structure for $\mathrm{NH}_{3}$ diffusion, the abundant active sites to adsorb $\mathrm{NH}_{3}$, and excellent conductivity for efficient electron transport, particularly the effect of RPcCo.

The effect of different RPcCos on the $\mathrm{NH}_{3}$ sensing properties of RPcCo/rGO hybrids was further studied by the first-principles density functional theory (DFT). Considering fewer interactions of $\mathrm{NH}_{3}$ and $\mathrm{rGO},{ }^{17}$ only the interactions between $\mathrm{NH}_{3}$ with the RPcCos were investigated. The calculation results are shown in 
Table 1 Calculated adsorption energies, major bond distance, and net charges in the adsorption of $\mathrm{NH}_{3}$ on the RPcCos

\begin{tabular}{llll}
\hline & & & \\
Sub. $^{a}$ & Net charge $^{b}$ & $d(\mathrm{Co}-\mathrm{N})^{c}(\AA)$ & $\begin{array}{l}\text { Adsorption energy } \\
\left(\mathrm{kcal} \mathrm{mol}^{-1}\right)\end{array}$ \\
\hline cpoPcCo & 0.189 & 2.193 & -23.1 \\
cmpoPcCo & 0.187 & 2.194 & -22.4 \\
poPcCo & 0.186 & 2.195 & -22.0 \\
mpoPcCo & 0.184 & 2.197 & -21.7 \\
${ }^{a}$ The substrate. & ${ }^{b}$ The net charge on $\mathrm{NH}_{3} \cdot{ }^{c}$ Distance between cobalt and \\
$\mathrm{NH}_{3}$. &
\end{tabular}

Table 1. The bond length of cpoPcCo/rGO $\left(r_{\mathrm{Co}-\mathrm{N}}, 2.193\right)$ was less than that of the other hybrids. The $r_{\mathrm{Co}-\mathrm{N}}$ parameter illustrates the adsorption interactions between $\mathrm{NH}_{3}$ and RPcCos. A smaller bond length means stronger adsorption between $\mathrm{NH}_{3}$ and RPcCos. It can be further confirmed by calculating the adsorption energy between the RPcCo- $\mathrm{NH}_{3}$ systems, which can be seen to be negative, indicating that the $\mathrm{NH}_{3}$ adsorption on the RPcCo complex is an exothermic process, which will facilitate the formation of a stable structure. ${ }^{43,49}$ The adsorption energy of cpoPcCo- $\mathrm{NH}_{3}\left(-23.1 \mathrm{kcal} \mathrm{mol}^{-1}\right)$ is more negative than that of the other hybrids, hence, $\mathrm{NH}_{3}$ shows affinity to interact on cpoPcCo/rGO. To elucidate the charge transfer between the $\mathrm{NH}_{3}$ and RPcCos, the NBO calculations were performed. In general, the net charges on $\mathrm{NH}_{3}$ are positive (Table 1), implying that there occurs electron transfer from $\mathrm{NH}_{3}$ to RPcCos. Charge transfer indicates a charge transfer sensing mechanism for the $\mathrm{RPcCo} / \mathrm{rGO}$ sensors, which agrees well with the experimental observation. It is noted that there is much charge transfer in the adsorption of $\mathrm{NH}_{3}$ on cpoPcCo $(0.189 \mathrm{eV})$ than that of other systems. In short, the $\mathrm{NH}_{3}$ sensitivity of cpoPcCo/rGO hybrid is the highest. Moreover, the cmpoPcCo/rGO hybrid shows also a better response than other two kinds of hybrids, further illustrating that the carboxyl substituent played a critical role in gas sensitivity. To further verify the charge transfer between RPcCos and $\mathrm{NH}_{3}$, the electrochemical impedance spectroscopy (EIS) of $\mathrm{RPcCo} / \mathrm{rGO}$ hybrids was performed (Fig. 6). Fig. S19† shows the EIS of rGO. The fitting EIS parameters of the rGO and $\mathrm{RPcCo} / \mathrm{rGO}$ hybrids were analyzed in Table 2 . The $R_{\mathrm{b}}$ and $R_{\mathrm{ct}}$ of the cpoPcCo/rGO $(16.61,18.74 \Omega)$ and cmpoPcCo/rGO $(16.92$,

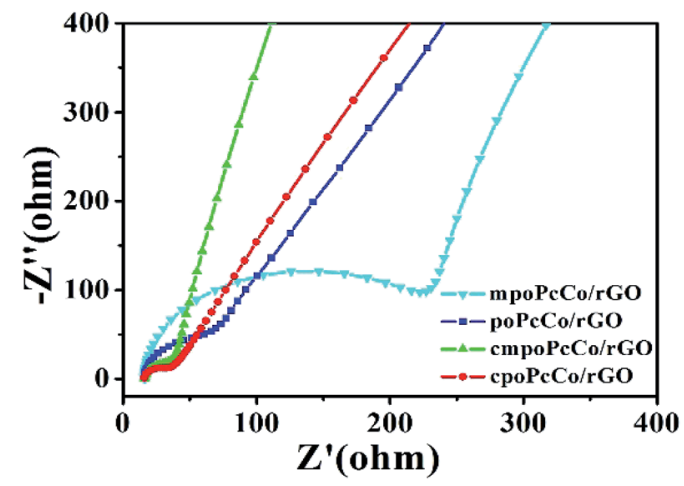

Fig. 6 Nyquist plots of RPcCo/rGO hybrids.
Table 2 Fitted impedance parameters of $\mathrm{rGO}$ and PcCo/rGO electrode

\begin{tabular}{lcr}
\hline Samples & $R_{\mathrm{b}}(\Omega)$ & $R_{\mathrm{ct}}(\Omega)$ \\
\hline cpoPcCo/rGO & 16.61 & 18.74 \\
cmpoPcCo/rGO & 16.92 & 27.58 \\
poPcCo/rGO & 18.30 & 234.15 \\
mpoPcCo/rGO & 19.03 & 381.32 \\
rGO & 28.53 & 785.80 \\
\hline
\end{tabular}

$27.58 \Omega$ ) hybrids are obviously lower than those of poPcCo/rGO (18.30, $234.15 \Omega)$, mpoPcCo/rGO (19.03, 381.32 $\Omega$ ) and rGO $(28.53,785.80 \Omega)$, respectively. $R_{\mathrm{b}}$ is the uncompensated resistance of the electrolyte, separator and electrode. $R_{\mathrm{ct}}$ is related to the electrical conductivity and electron transport of the material. The lower the values of $R_{\mathrm{b}}$ and $R_{\mathrm{ct}}$, the stronger the electron transport capability. Therefore, the $\mathrm{cpoPcCo} / \mathrm{rGO}$ and cmpoPcCo/rGO hybrids exhibit a superior response to $\mathrm{NH}_{3}$ over the other hybrids, which is consistent with the DFT analysis.

\section{Conclusions}

In summary, RPcCo/rGO hybrids were successfully prepared by coating four different phenoxyl substituents PcCos on the surface of rGO via non-covalent interactions, which is advantageous for maintaining the structural superiority of rGOs as well as the active sites for the $\mathrm{NH}_{3}$ adsorption by PcCos. Due to the large-surface-area structure for $\mathrm{NH}_{3}$ diffusion, the abundant active sites to adsorb $\mathrm{NH}_{3}$, and excellent conductivity for efficient electron transport, the obtained sensors exhibited excellent sensitivity, selectivity, good reproducibility and perfect response-concentration linearity to $\mathrm{NH}_{3}$ at room temperature. In particular, the cpoPcCo/rGO based sensor shows a high and fast response for a low concentration of $\mathrm{NH}_{3}\left(\sim 2.5\right.$ and $45 \mathrm{~s}$ for $100 \mathrm{ppb}$ of $\left.\mathrm{NH}_{3}\right)$, a ppb level detection and superior stability over 60 days. Besides, the sensitivity order of $\mathrm{PcCo} / \mathrm{rGO}$ hybrids to $\mathrm{NH}_{3}$ is cpoPcCo/rGO > cmpoPcCo/rGO > poPcCo/rGO > mpoPcCo/rGO, and is supported by the first-principles density functional theory and impedance. Therefore, it is a valid pathway to design the functionalized rGO-based gas-sensing materials by regulating the substituent groups of RPcCo.

\section{Conflicts of interest}

There are no conflicts to declare.

\section{Acknowledgements}

We are grateful for the financial support from the National Natural Science Foundation of China (51202061), the Natural Science Foundation of Heilongiiang Province of China (LH2019E076), the Innovative Talents Program of Harbin (2017RAQXJ143). 


\section{References}

1 H. F. Fei, G. Wu, W. Y. Cheng, W. J. Yan, H. J. Xu, D. Zhang, Y. F. Zhao, Y. H. Lv, Y. H. Chen, L. Zhang, C. Ó. Coileain, C. L. Heng, C. R. Chang and H. C. Wu, ACS Omega, 2019, 4, 3812-3819.

2 M. R. U. D. Biswas and W. C. Oh, RSC Adv., 2019, 9, 1148411492.

3 S. Q. Li, Y. J. Diao, Z. J. Yang, J. M. He, J. Wang, C. C. Liu, F. M. Liu, H. Y. Lu, X. Yan, P. Sun and G. Y. Lu, Sens. Actuators, B, 2018, 276, 526-533.

4 L. S. K. Acharya, A. Kumara, B. Barika, P. S. Nayaka, N. Tripathyb, J. P. Karb and P. Dasha, Sens. Actuators, B, 2018, 272, 100-109.

5 J. Ma, X. Y. Guo, Y. P. Ying, D. H. Liu and C. L. Zhong, Chem. Eng. J., 2017, 313, 890-898.

6 B. Ramezanzadeh, Z. Haeri and M. Ramezanzadeh, Chem. Eng. J., 2016, 303, 511-528.

7 K. S. Novoselov, A. K. Geim, S. V. Morozov, D. Jiang, Y. Zhang, S. V. Dubonos, I. V. Grigorieva and A. A. Firsov, Science, 2004, 306, 666-669.

8 F. Schedin, A. K. Geim, S. V. Morozov, E. W. Hill, P. Blake, M. I. Katsnelson and K. S. Novoselov, Nat. Mater., 2007, 6, 652-655.

9 T. O. Wehling, K. S. Novoselov, S. V. Morozov, E. E. Vdovin, M. I. Katsnelson, A. K. Geim and A. I. Lichtenstein, Nano Lett., 2008, 8, 173-177.

10 O. Leenaerts, B. Partoens and F. M. Peeters, Phys. Rev. B: Condens. Matter Mater. Phys., 2008, 77, 125416-125422.

11 G. Chen, T. M. Paronyan and A. R. Harutyunyan, Appl. Phys. Lett., 2012, 101, 053119.

12 W. W. Li, X. Li, L. Cai, Y. L. Sun, M. X. Sun and D. Xie, J. Nanosci. Nanotechnol., 2018, 18, 7927-7932.

13 Y. Zhou, X. G. Lin, Y. K. Huang, Y. C. Guo, C. Gao, G. Z. Xie and Y. D. Jiang, Sens. Actuators, B, 2016, 235, 241-250.

14 G. H. Lu, S. J. Park, K. H. Yu, R. S. Ruoff, L. E. Ocola, D. Rosenmann and J. H. Chen, ACS Nano, 2011, 5, 11541164.

15 M. J. Allen, V. C. Tung and R. B. Kaner, Chem. Rev., 2010, 110, 132-145.

16 S. Mao, S. M. Cui, G. H. Lu, K. H. Yu, Z. H. Wen and J. H. Chen, J. Mater. Chem., 2012, 22, 11009-11013.

17 W. J. Yuan and G. Q. Shi, J. Mater. Chem. A, 2013, 1, 1007810091.

18 M. Hakimi, A. Salehi, F. A. Boroumand and N. Mosleh, IEEE Sens. J., 2018, 18, 2245-2252.

19 I. Karaduman, E. Er, H. Celikkan, N. Erk and S. Acar, J. Alloys Compd., 2017, 722, 569-578.

20 Z. B. Ye, H. L. Tai, R. Guo, Z. Yuan, C. H. Liu, Y. J. Su, Z. Chen and Y. D. Jiang, Appl. Surf. Sci., 2017, 419, 84-90.

21 Q. Q. Sun, W. G. Feng, P. Yang, G. Q. You and Y. L. Chen, New J. Chem., 2018, 42, 6713-6718.

22 R. P. Linstead, J. Chem. Soc., 1934, 1016-1017.

23 T. Sizun, M. Bouvet and J. M. Suisse, Talanta, 2012, 97, 318324.
24 C. E. Dent, R. P. Linstead and A. R. Lowe, J. Chem. Soc., 1934, 1033-1039.

25 H. Ma, H. X. Zhang, M. Q. Tong, J. D. Cao and W. Wu, RSC Adv. , 2019, 9, 24751-24759.

26 J. Zhang, X. H. Liu, G. Neri and N. Pinna, Adv. Mater., 2016, 28, 795-831.

27 X. L. Huang, N. T. Hu, R. G. Gao, Y. Yu, Y. Y. Wang, Z. Yang, E. S. W. Kong, H. Wei and Y. F. Zhang, J. Mater. Chem., 2012, 22, 22488-22495.

28 B. Wang, X. L. Wang, X. C. Li, Z. J. Guo, X. Zhou and Y. Q. Wu, RSC Adv., 2018, 8, 41280-41287.

29 X. Q. Zhou, X. L. Wang, B. Wang, Z. M. Chen, C. Y. He and Y. Q. Wu, Sens. Actuators, B, 2014, 193, 340-348.

30 H. Wu, Z. M. Chen, J. L. Zhang, F. Wu, C. Y. He, B. Wang, Y. Q. Wu and Z. Y. Ren, J. Mater. Chem. A, 2016, 4, 1096-1104.

31 B. Wang, X. Q. Zhou, Y. Q. Wu, Z. M. Chen and C. Y. He, Sens. Actuators, B, 2012, 171-172, 398-404.

32 T. Yanaia, D. P. Tew and N. C. Handyb, Chem. Phys. Lett., 2004, 393, 51-57.

33 E. D. Glendening, A. E. Carpenter and F. Weinhold, NBO, version 3.1, 1995.

34 M. J. Frisch, et al., Gaussian 09, Revision B01, Gaussian, Inc., Wallingford, CT, 2009.

35 A. Günsel, A. T. Bilgiçli, H. Pişkin, N. Ç. Delibaş, M. N. Yarasir and B. Gündüz, New J. Chem., 2018, 42, 6013-6022.

36 H. Z. Zhao, Y. Zhang, B. Zhao, Y. Y. Chang and Z. S. Li, Environ. Sci. Technol., 2012, 46, 5198-5204.

37 F. D. Souza and O. Ito, Chem. Commun., 2009, 4913-4928.

38 S. Harbeck, O. F. Emirik, I. Gurol, A. G. Gurek, Z. Z. Ozturk and V. Ahsen, Sens. Actuators, B, 2013, 176, 838-849.

39 H. Wu, Z. M. Chen, J. L. Zhang, F. Wu, C. H. He, Z. Y. Ren and Y. Q. Wu, Chem. Mater., 2017, 29, 9509-9517.

40 A. Chunder, T. Pal, S. I. Khondaker and L. Zhai, J. Phys. Chem. C, 2010, 114, 15129-15135.

41 J. H. Zhu, Y. X. Li, Y. Chen, J. Wang, B. Zhang and J. J. Zhang, Carbon, 2011, 49, 1900-1905.

42 X. L. Huang, N. T. Hu, R. G. Gao, Y. Yu, Y. Y. Wang, Z. Yang, E. S. W. Kong, H. Weia and Y. F. Zhang, J. Mater. Chem., 2012, 22, 22488-22495.

43 Y. Li, B. Wang, Z. Y. Yu, X. Q. Zhou, D. Kang, Y. Q. Wu, Z. M. Chen, C. Y. He and X. Zhou, RSC Adv., 2017, 7, 34215-34225.

44 S. Mao, G. H. Lu and J. H. Chen, J. Mater. Chem. A, 2014, 2, 5573-5579.

45 F. I. Bohrer, C. N. Colesniuc, J. Park, M. E. Ruidiaz, I. K. Schuller, A. C. Kummel and W. C. Trogler, J. Am. Chem. Soc., 2009, 131, 478-485.

46 M. Manouchehrian, M. M. Larijani and S. M. Elahi, Mater. Res. Bull., 2015, 62, 177-183.

47 H. Liu, M. Li, O. Voznyy, L. Hu, Q. Y. Fu, D. X. Zhou, Z. Xia, E. H. Sargent and J. Tang, Adv. Mater., 2014, 26, 2718-2724.

48 Y. L. Liu, H. R. Wang, K. Q. Chen, T. Q. Yang, S. Yang and W. Chen, ACS Appl. Mater. Interfaces, 2019, 11, 9573-9582.

49 Z. F. Cao, Q. B. Chen, Y. X. Lu, H. L. Liu and Y. Hu, Int. J. Quantum Chem., 2013, 113, 1137-1146. 